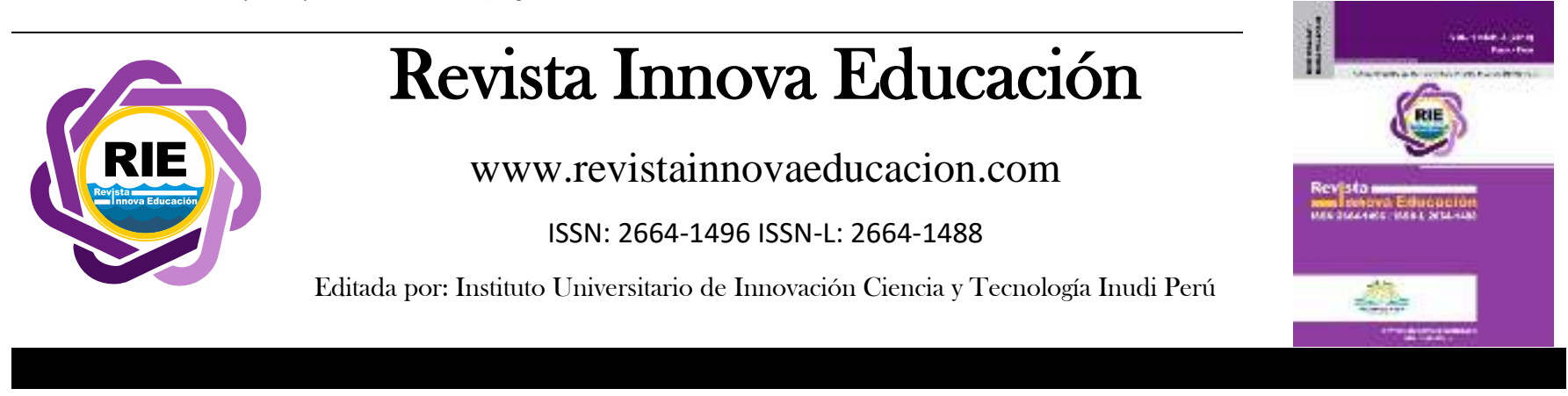

\title{
Educación tributaria y recaudación fiscal de rentas de primera categoría en estudiantes universitarios del Perú
}

\author{
Tax education and first category income tax collection in Peru vian university students \\ Edgar Callohuanca ${ }^{1}$ \\ Universidad Nacional del Altiplano de Puno, Puno-Puno, Perú \\ (D) https://orcid.org/0000-0001-8463-5684 \\ Ludwing Flores \\ Universidad Nacional del Altiplano de Puno, Puno-Puno, Perú \\ (D) https://orcid.org/0000-0002-2946-8710 \\ Wilson Sucari \\ Universidad Nacional del Altiplano de Puno, Puno-Puno, Perú \\ (D) https://orcid.org/0000-0001-5874-0966
}

DOI: https://doi.org/10.35622/j.rie.2020.03.009

Recibido 23/03/2020/ Aceptado 30/07/2020 Publicado 30/07/2020

\begin{tabular}{|c|c|}
\hline ARTÍCULO ORIGINAL & $\mathrm{rel}$ \\
\hline PALABRAS CLAVE & recaudación fiscal de rentas de primera categoría en estudiantes universitarios del Perú. \\
\hline $\begin{array}{l}\text { Educación tributaria, } \\
\text { estudiantes, } \\
\text { recaudación }\end{array}$ & $\begin{array}{l}\text { Este trabajo se ubica en el enfoque cuantitativo, de tipo descriptivo y de diseño } \\
\text { correlacional transversal. La muestra estuvo conformada por } 3560 \text { estudiantes (entre } \\
\text { varones y mujeres de } 18 \text { a } 25 \text { años), el cual se obtuvo mediante la técnica de muestreo } \\
\text { no probabilístico y por conveniencia. Para la obtención de datos se aplicó el }\end{array}$ \\
\hline tributaria, universidad & $\begin{array}{l}\text { Cuestionario de Educación Fiscal, el cual fue elaborado por los autores bajo los } \\
\text { presupuestos teóricos del Tribunal Fiscal adscrito al Ministerio de Economía y Finanzas } \\
\text { del Perú. El procesamiento estadístico se realizó mediante la técnica de análisis de } \\
\text { varianza (ANOVA) de las variables educación tributaria (1) y recaudación fiscal (2). } \\
\text { Los resultados indicaron que existe asociación directa entre las variables mencionadas } \\
\text { con un p-valor }=0.001 \text {. En conclusión, la educación tributaria se relaciona de manera } \\
\text { directa con la recaudación fiscal. }\end{array}$ \\
\hline
\end{tabular}

\section{KEYWORDS}

Tax education, students, tax collection, university
ABSTRACT. The objective was to evaluate the level of tax education related to the first category income tax collection in Peruvian university students. This work is in the quantitative approach, descriptive type, and cross-correlational design. The sample consisted of 3,560 students (between men and women between 18 and 25 years old), which was obtained using the non-probabilistic and convenience sampling technique. To obtain data, the Tax Education Questionnaire was applied, which was prepared by

\footnotetext{
${ }^{1}$ Correspondencia: ecallohuanca@unap.edu.pe
} 
the authors under the theoretical assumptions of the Tax Court attached to the Ministry of Economy and Finance of Peru. The statistical processing was carried out using the variance analysis technique (ANOVA) of the variables tax education (1) and tax collection (2). The results indicated that there is a direct association between the mentioned variables with a p-value $=0.001$. In conclusion, tax education is directly related to tax collection.

\section{INTRODUCCIÓN}

Es necesario dar a conocer las normas, procedimientos y principios al momento de alquilar un inmueble y de esta manera tener un alto nivel de recaudación tributaria (Toledo, Román y Nistal 1999). Los arrendadores aparentemente son renuentes a cumplir con sus obligaciones tributarias, incurriendo en evasión de los tributos (Davis \& González, 1998). Lo cual se convierte en un gran problema que ocasiona la disminución de la recaudación de los ingresos fiscales, que sirven para financiar las actividades que realiza el Estado en favor de las áreas más importantes de la población (Sánchez \& Silva 2018). Es producto básicamente de la inquietud por conocer el cumplimiento de las normas legales, respecto de los ingresos por alquileres de inmuebles que tienen muchos estudiantes universitarios de nuestro País, especialmente en barrios aledaños a las universidades, que concentra gran cantidad de estudiantes que son inmigrantes de distintas provincias; la investigación denota importancia porque los ingresos de renta de primera categoría no es cumplida por los arrendatarios y ello ocasiona evasión tributaria que perjudica al fisco, esta situación muestra la deficiente educación tributaria en el arrendamiento de inmuebles.

La evasión tributaria significa la restricción del gasto público y la ejecución deficiente de los planes de diferentes gobiernos, tales como son: el tributo, es falta de cumplimiento puede derivar en pérdida efectiva de ingreso para el fisco o no, la evasión tributaria es definida como la falta de cumplimiento de sus obligaciones por parte de los contribuyentes (Morales, 2019). La obligación tributaria es la relación que existe entre el acreedor y deudor tributario originado por el tributo, establecido en la ley (Ramos \& Morales, 2019), en la misma línea es el de derecho público que representa la relación que existe entre deudor y el acreedor tributario (Arce \& Vargas 2010), es también el vínculo jurídico originado por el tributo, cuya fuente es la ley, y constituye en nexo entre el estado (acreedor) y el responsable o contribuyente (deudor), que se manifiesta en una obligación de dar (Zapata \& Rodríguez, 2017). En relación con la renta como tributo no vinculado constituye una de las principales fuentes de recurso del estado (Martínez, 2009), es la utilidad o ganancia susceptible, producto periódico que proviene de una fuente durable en estado de explotación (López \& Bonilla 2008). 
En relación a rentas de primera categoría son las rentas de la explotación de predios y bienes muebles, siempre que no estén expresamente considerados como rentas de segunda categoría, comprende las rentas producidas por el arrendamiento, subarrendamiento y cesión de bienes (Sánchez \& Hernández, 2018). El producto en efectivo o en especie del arrendamiento o subarrendamiento de predios, incluidos sus accesorios, así como es importe pactado por los servicios suministrados por el locador y el monto de los tributos que tome a su cargo el arrendatario y legalmente corresponda al locador (Chávez, 2018). En esta misma línea se considera el valor de mejoras de predios: el valor de mejoras introducidas de predios por el arrendador o su arrendatario, en tanto constituyan un beneficio para el propietario y en la parte que este no se encuentre obligado a reembolsar (Gantman, 2011), como también precios cedidos gratuitamente: La renta ficta de predios cuya ocupación hayan cedido sus propietarios gratuitamente o a precio no determinado (SUNAT, 2013), también la renta neta de primera categoría se establece la manera conjunta con la renta de segunda categoría aplicando una deducción global del 20\% (López y Bonilla 2008).

En relación con los impuestos cuyo cumplimiento no influye una contraprestación directa en favor del contribuyente por parte del estado. Es unánime la doctrina al señalar que la característica primordial de los impuestos es que no existe relación entre los que se paga y el cargo de estos recursos, mencionado, por ese motivo, como tributos no vinculados, por cuanto su exigibilidad es independiente de cualquier actividad estatal y/o privada referida al contribuyente (MEF, 2017), el impuesto está compuesto por: impuesto directo; es aquel que incide directamente sobre el ingreso o patrimonio de las personas naturales y jurídicas e impuesto indirecto. aquel que transgrede sobre el consumo, pero quien lo paga es el consumidor del producto o servicio (Sánchez, 2018).

En ese sentido, este trabajo de investigación se planteó las siguientes interrogantes: ¿cómo es el nivel de educación tributaria? ¿Cómo es el nivel de recaudación tributaria? ¿Como es el nivel de asociación de la educación tributaria y recaudación fiscal?

\section{MÉTODO Y MATERIALES}

\section{Objetivos}

- Identificar el nivel de educación tributaria.

- Identificar el nivel de recaudación fiscal.

- Determinar la asociación entre la educación tributaria y la recaudación fiscal.

\section{Método y diseño de investigación}


Este trabajo corresponde al método hipotético deductivo, de tipo descriptivo y de diseño correlacional transversal.

\section{Variables}

- Educación tributaria: Abarca las dimensiones de cultura y conocimiento tributarios.

- Recaudación Fiscal: Abarca las dimensiones de obligaciones formales y obligaciones sustanciales.

\section{Muestra}

Se trabajó mediante la técnica de muestreo no probabilístico por conveniencia con un número de 3560 estudiantes. Participaron principalmente estudiantes de las regiones de Lima, Puno y Piura.

\section{Instrumentos}

Se aplicó un cuestionario de tipo cerrado, estructurado en dimensiones y establecido en la escala de Likert (1=nunca y 5=siempre) para determinar los niveles de Educación. Este cuestionario fue elaborado por los autores de esta investigación bajo los fundamentos teóricos y normativos del Tribunal Fiscal adscrito al Ministerio de Economía y Finanzas del Perú (20192020). Para determinar la fiabilidad del instrumento, previamente, se aplicó una prueba piloto a 112 estudiantes universitarios, teniendo una confiabilidad de 0.712 en el coeficiente de Alfa de Cronbach.

Por otro lado, los niveles de recaudación tributaria resultaron de la sistematización de la base de datos de la Superintendencia Nacional de Aduanas y Administración Tributaria (SUNAT, 2019-2020) en el impuesto de rentas de primera categoría.

\section{Diseño estadístico}

Para el procesamiento estadístico se realizó mediante la técnica de análisis de varianza (ANOVA) de las variables educación tributaria (1) y recaudación fiscal (2).

H0: No hay diferencias entre las medias de los diferentes grupos: $\mu 1=\mu 2 \ldots=\mu \mathrm{k}=\mu$ H1: Al menos un par de medias son significativamente distintas la una de la otra.

\section{RESULTADOS}

\subsection{Niveles de educación tributaria}


Respondieron 3560 estudiantes universitarios a la totalidad de las preguntas formuladas a través del Formulario de Google. Según el estudio se pudo determinar que existe un bajo nivel educación tributaria (83.85\%), este resultado es desfavorable para las políticas tributarias que se vienen implementando en el Perú. Por otro lado, la educación tributaria es la base para la recaudación y el sostenimiento del país, este indicador encierra un conjunto de valores, creencias y actitudes compartidas por una sociedad respecto a la tributación y a la observancia de las leyes. Esto va de la mano con la confianza y credibilidad al gobierno.

Tabla 1. Niveles de educación tributaria de estudiantes universitarios del Perú, 2020

\begin{tabular}{ccc}
\hline Niveles & Cantidad & Porcentaje \\
\hline Alto & 215 & $6,04 \%$ \\
Regular & 360 & $10,11 \%$ \\
Bajo & 2985 & $83,85 \%$ \\
\hline Total & $\mathbf{3 5 6 0}$ & $\mathbf{1 0 0 , 0 0 \%}$ \\
\hline
\end{tabular}

Fuente: Sistematización de datos del Cuestionario de Educación Fiscal

\subsection{Niveles de recaudación fiscal}

De los resultados se confirma que el $87.02 \%$ tiene un bajo cumplimiento de las obligaciones formales y sustanciales del impuesto a la renta de primera categoría. Por ende, la SUNAT no tiene presencia en dicha categoría de recaudación, lo que genera una serie de perjuicios al fisco.

Tabla 2. Niveles de recaudación tributaria de estudiantes universitarios del Perú

\begin{tabular}{lll}
\hline Niveles & Cantidad & Porcentaje \\
\hline Alto & 150 & $4,21 \%$ \\
Regular & 312 & $8,76 \%$ \\
Bajo & 3098 & $87,02 \%$ \\
\hline Total & $\mathbf{3 5 6 0}$ & $100,00 \%$ \\
\hline
\end{tabular}

Fuente: Base de datos de la Superintendencia Nacional de Aduanas y Administración Tributaria

\subsection{Correlación entre educación tributaria y recaudación fiscal}

Tabla 3. Tabla Cruzada de recaudación y educación tributarias

\begin{tabular}{lrrrrrrrr}
\multicolumn{7}{c}{ Recaudación Tributaria } \\
\cline { 3 - 9 } & 1,00 & \multicolumn{1}{c}{, 00} & 1,00 & 2,00 & 3,00 & 4,00 & 5,00 & \multicolumn{1}{c}{ Total } \\
& 2,00 & 0 & 46 & 783 & 0 & 0 & 9 & 838 \\
Educación & 3,00 & 0 & 48 & 0 & 782 & 0 & 8 & 838 \\
Tributaria & 4,00 & 0 & 42 & 0 & 195 & 392 & 208 & 837 \\
& 5,00 & 0 & 21 & 0 & 0 & 0 & 398 & 419
\end{tabular}




\begin{tabular}{llllllll}
\hline Total & 398 & 189 & 977 & 977 & 392 & 627 & 3560 \\
\hline
\end{tabular}

Fuente: IBM SPSS Statistics 25

Conforme a la tabla 3 se aprecia que existe un alto nivel de significancia en una escala vigesimal de la educación tributaria y la recaudación determinando que, según Ramos y Morales (2019), la educación tributaria se identifica con el cumplimiento voluntario de los deberes y obligaciones tributarios por parte del contribuyente y no con la implementación de estrategias para incrementar la recaudación de tributos bajo presión, por temor a las sanciones. Se evidencia una desinformación acerca de las estrategias y planes que adelanta la administración tributaria en pos de educar al contribuyente (Moreno y Uribe, 2010). Entre las conclusiones más importantes se señalan las estrategias y programas educativos utilizados por la SUNAT para desarrollar la educación tributaria del contribuyente. Con la educación tributaria se pretende que los individuos de la sociedad involucrados en el proceso tomen conciencia en cuanto al hecho de que es un deber constitucional aportar al Estado y comunicar a esa colectividad que las razones fundamentales de la tributación son el proporcionar a la Nación los medios necesarios para que este cumpla con su función primaria como lo es la de garantizar a la ciudadanía servicios públicos eficaces y eficientes.

Tabla 4. Anova de la recaudación y educación tributaria

ANOVA

\begin{tabular}{lccrrrr}
\hline & $\begin{array}{c}\text { Suma de } \\
\text { cuadrados }\end{array}$ & gl & Media cuadrática & F & Sig. \\
\hline Entre grupos & 5958.397 & & 4 & 1489.599 & 2611.77 & 0.001 \\
& & & & & 7 & \\
Dentro de grupos & 2027.556 & 3555 & 0.570 & & \\
Total & 7985.953 & 3559 & & & \\
\hline
\end{tabular}

Fuente: IBM SPSS Statistics 25

Como se observa en la tabla 4 se tiene una significativa relación entre la recaudación con la cultura tributaria $(F=2611.777$ y P = 0,001). Hernando de Soto (2006) sostiene que en el Perú el problema no está en la economía informal, sino en el Estado. "Aquella es, más bien, una respuesta popular espontánea y creativa ante la incapacidad estatal para satisfacer las aspiraciones más elementales de los pobres. Cuando la legalidad es un privilegio al que solo se accede mediante el poder económico y político, a las clases populares no les queda otra alternativa que la ilegalidad" (Hernando de Soto, 2006, p. 55). 


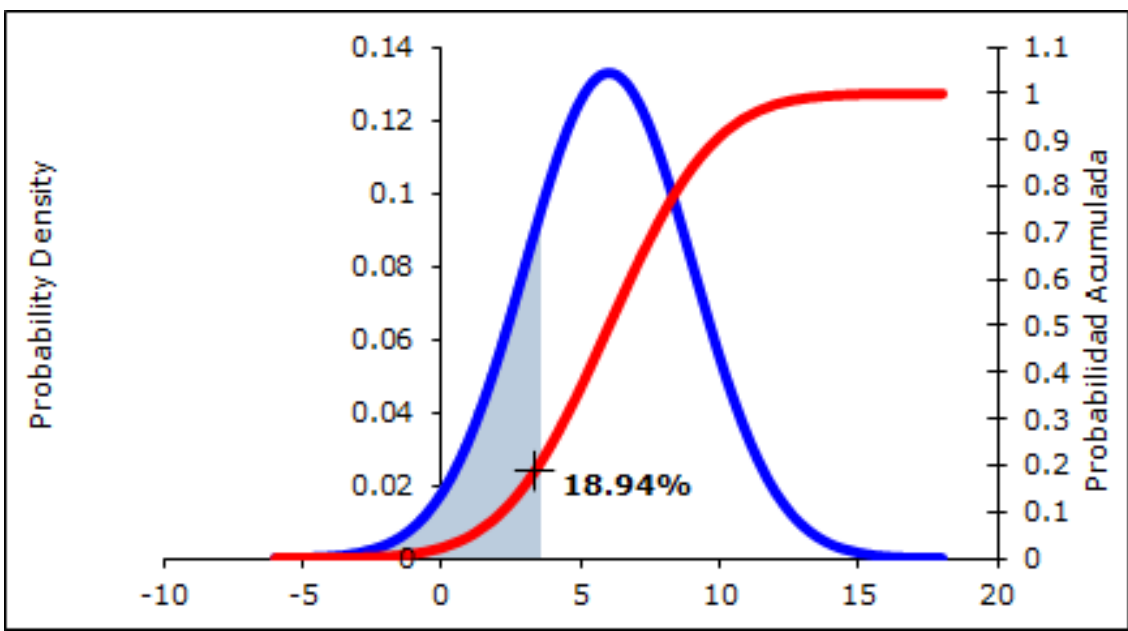

Figura 1. Prueba de normalidad de la recaudación tributaria del impuesto a la renta de primera categoría.

Conforme a la figura 1 , se tiene que todas las dimensiones de la variable recaudación tributaria presentan una distribución normal respecto a la misma varianza, lo cual les otorga mayor solidez a los cuadros precedentes. En razón a estos resultados, se proporciona una ganancia mayor sobre las aportaciones efectuadas por los grupos de interés respecto a la óptima educación tributaria y se mantiene la adecuada recaudación tributaria. La política tributaria no está acorde con la realidad de cada contexto los cuales procuran privilegiar a unos cuantos grupos de interés generando desconfianza de contribución en la población.

\section{DISCUSIONES}

Los estudios recientes reflejan un vínculo directo entre la ética fiscal y la percepción de los ciudadanos tanto en materia tributaria como acerca de la calidad del gasto público (Gil y García, 2014). Se aprecia una mayor predisposición para pagar impuestos entre aquellos segmentos sociales que perciben una mayor calidad de los servicios públicos prestados (Armas y Ramírez, 2016). Esta situación de escasa ética fiscal no sólo debilita los sentimientos de pertenencia a un proyecto social común, sino que pone de manifiesto la existencia de un círculo vicioso en virtud del que la evasión fiscal o la negativa a pagar más impuestos se justifica bajo el pretexto de que el Estado no cumple su parte del contrato social, lo que conduce al mismo tiempo a que los servicios 
no puedan mejorar por ser insuficientes los ingresos, de ahí que los servicios se deterioren, degeneran y el ciclo se perpetúe (López \& Bonilla, 2008).

Las Administraciones Tributarias de América Latina y del mundo han visto que la solución a los problemas económicos y el desarrollo de los pueblos está en la educación tributaria; considerándose desde un punto de vista social con la obtención de valores éticos y morales, a través de una convivencia ciudadana que dan base y legitimidad social a la tributación y al cumplimiento de las obligaciones tributarias como una necesidad del país, siendo el Estado el interesado de promover este proyecto (Leiva \& Vargas, 2019). A grandes rasgos ese suele ser el entorno que, en buena parte de América Latina, encuentran las administraciones tributarias para desarrollar sus funciones de educación fiscal (Jiménez \& Jacinto, 2010). Sin embargo y desde un punto de vista positivo, se trata también de campos que pueden ser fértiles para la siembra y el cultivo de los valores éticos y de convivencia ciudadana que dan base y legitimidad social a la tributación y al cumplimiento de las obligaciones tributarias como una necesidad de país (Chávez, 2018), para hacer viable el desarrollo económico, social y político que el Estado tiene por misión promover (Sánchez \& Fernández, 2018).

Esa perspectiva permite visualizar la amplitud del territorio sociocultural que se presenta a las iniciativas para la promoción de educación tributaria y que les plantea, desde su concepción, la necesidad de establecer prioridades, elegir grupos objetivo, fijar metas y definir modos de abordaje consistentes con la estrategia institucional de la administración tributaria y los recursos disponibles para tal fin (Moreno y Uribe, 2010).

La falta de educación tributaria lleva a la evasión tributaria, lo que genera retraso para el país (Arce, 2010). Para que el Estado pueda cumplir con su obligación constitucional de velar por el bien común y proporcionar a la población los servicios básicos que ésta requiere, necesita de recursos que provienen principalmente de los tributos pagados por los contribuyentes (Castillo y Guerra, 2018). Es importante que todos los ciudadanos posean una fuerte educación tributaria para que puedan comprender que los tributos son recursos que recauda el Estado en carácter de administrador, pero en realidad esos recursos le pertenecen a la población, por lo tanto, el Estado se los debe devolver prestando servicios públicos (como por ejemplo los hospitales, colegios, entre otros) (Mejía, 2014).

El cumplimiento tributario voluntario va relacionado con la moral fiscal que está constituida por una sola variable denominada tolerancia al fraude inscrita en la dimensión de los valores y motivaciones internas del individuo (Ortiz Celis, 2015); los resultados por el 
incumplimiento de las Administraciones Tributarias, en las cuales existen altas percepciones de riesgo así como la sensibilidad al aumento de las sanciones, no tienen relación con la gran magnitud del incumplimiento de las obligaciones tributarias (Wulff, 2008). Por este motivo se aborda un breve análisis de la situación tributaria, así como del cumplimiento tributario y la evasión tributaria en el Perú (Rosero, 2011), realizado mediante estudios en base de encuestas sobre Conciencia Tributaria, obteniendo resultados de un enfoque que sirve para formular un nuevo marco teórico y analítico acerca de la moral fiscal de los peruanos en el que queda demostrado que los valores personales e incluso la visión que tienen los ciudadanos en relación con el Estado (Jiménez \& Jacinto, 2010b), tienen una pobre contribución pues el proceso de internalización del sistema legal no ha concluido, es incipiente donde prima la norma social que ha sido generada por una democracia imperfecta que no ha permitido el cumplimiento del contrato social, generando inequidades fiscales importantes (Martínez \& Maldonado, 2016).

El hecho tributario es un acto de dos vías entre el contribuyente y el Estado, en el que a este último corresponde una doble función: la de recaudar los impuestos y la de retornarlos a la sociedad bajo la forma de bienes y servicios públicos. Se trata de un proceso social regulado, en el cual el cumplimiento tributario responde a un mandato legal que el contribuyente debe acatar y que la autoridad debe hacer cumplir, según las facultades que la ley le confiere (Sandoval et al., 2017). De ahí que la conciencia tributaria de los ciudadanos se pueda fortalecer al aplicar mecanismos de control más estrictos, siempre que el Estado dé muestras de administración honesta y eficiente. Independientemente de que una sociedad se encuentre regida o no por un sistema democrático, el cumplimiento de los deberes fiscales responde a un orden legislado, provisto de normas, plazos y sanciones determinados, que dota a la autoridad tributaria de la potestad de cobrar los impuestos y de actuar en contra de quienes incumplan la obligación de pagarlos (Gavilánez, 2012). Sin embargo, el ámbito de acción de la Administración Tributaria va más allá del mero cobro de impuestos (Borja \& Pereira, 2020). Esto es algo que a muchas administraciones tributarias no les resulta tan evidente, algunas ven a la educación fiscal como algo superfluo o, en el mejor de los casos, como un elemento accesorio o complementario de sus quehaceres principales, sin percatarse que la formación de la educación tributaria representa, en el largo plazo, una de las bases más sólidas y confiables en las que puede sustentarse la recaudación (Gantman, 2011).

En ese sentido se propone que la SUNAT implante un sistema educativo de Cultura Tributaria a través de la capacitación de docentes y estos a su vez impartan sus conocimientos en los centros educativos como es inicial, primaria y secundaria. La capacitación de docentes es un 
programa de mediano y largo plazo, que permitirá desarrollar en los escolares conciencia sobre la importancia del cumplimiento de las obligaciones tributarias, como zona del rol ciudadano en una sociedad democrática.

\section{CONCLUSIONES}

El nivel de educación tributaria y el nivel de recaudación fiscal de los estudiantes universitarios del Perú son bajas (83.85\% y 87.02\%, respectivamente), los cuales explican que existen baja formación académica en temas tributarios en las universidades del Perú.

Por otro lado, la asociación entre la educación tributaria y recaudación fiscal es directa y significativa $(\mathrm{F}=2611.777$ con $\mathrm{P}$-valor $=0.001)$, el cual explica que si se toma en cuenta la formación de la educación tributaria muy probablemente el Estado peruano podrá recaudar en la misma relación mayores tributos.

\section{REFERENCIAS BIBLIOGRÁFICAS}

Arce, J. (2010). Importancia de establecer un acuerdo para evitar la Doble Tributación MéxicoEspaña. Universidad Autónoma de Estado de Hidalgo. https://www.uaeh.edu.mx/investigacion/productos/1689/

Armas, L. S. (2016). Tratamiento de los tributos ambientales en el sistema jurídico latinoamericano y peruano y el respeto de los principios preventivos y precautorios. Tesis para optar el Título Profesional de Abogado. Carrera de Derecho. Lima, Perú: Universidad Autónoma del Perú. 95 p.

Borja, F. M. B., \& Pereira, J. R. O. (2020). Factores que determinan la evasión del impuesto a la renta: Una revisión de la literatura. Interconectando Saberes, 9.

Castillo, D. (2018). Caracterización de los tributos aplicados a las empresas hoteleras en el Perú: Caso Los Gavilanes Hotel SRL-Pucallpa, 2017. Universidad Católica Los Ángeles de Chimbote. http://repositorio.uladech.edu.pe/handle/123456789/5862

Chávez, L. (2018). La NIIF y tributación: Desafíos y oportunidades para las administraciones tributarias. Revista internacional Legis de contabilidad \& auditoría, 74, 81-122.

Cortez, R., \& Orejuela, S. (2015). Análisis de la evasión tributaria del impuesto a la renta y su incidencia en los niveles de recaudación fiscal en el Cantón Milagro para el periodo 20132014 [B.S. thesis].

Gantman, E. (2011). La productividad científica argentina en ciencias sociales: Economía, psicología, sociología y ciencia política en el Conicet (2004-2008). Revista Española de Documentación Científica, 34(3), 408-425. 
Gavilánez, J. (2012). La universidad en la cultura tributaria. RETOS. Revista de Ciencias de la $\begin{array}{llll}\text { Administración } & y & \text { Economía, } & \text { 2(3), }\end{array}$ https://www.redalyc.org/articulo.oa?id=504550953007

Gil, E. (2014). Un nuevo modelo de tributación como medio para alcanzar la sostenibilidad energética. Revista de doctrina, legislación y jurisprudencia, 30(1) 11-42.

Jiménez, R. (2010). Evasión de impuestos nacionales en Colombia: Años 2001 - 2009. Revista de la Facultad de Ciencias Económicas: Investigación y Reflexión, XVIII (2), 177-198. https://www.redalyc.org/articulo.oa?id=90920053010

Juárez, O. (2010). Evasión de impuestos nacionales en Colombia: Años 2001 - 2009. Revista de la Facultad de Ciencias Económicas: Investigación y Reflexión, XVIII (2), 177-198. https://www.redalyc.org/articulo.oa?id=90920053010

Leiva, N. \& Marín, Y. (2019). La educación tributaria y su relación con la evasión tributaria en latinoamerica y el Perú, años 2012 al 2018. Universidad Privada del Norte. https://repositorio.upn.edu.pe/handle/11537/21013

Lejeune, E. (2014). Las reformas tributarias en España: Algunas reflexiones sobre cuestiones olvidadas. CEU Ediciones. https://repositorioinstitucional.ceu.es/handle/10637/6481

López, J., López, L. (2008). Producción científica española en marketing turístico. Estudios turísticos, 177, 35-50.

Martínez, M. P. V., \& Maldonado, J. C. (2016). Evasión fiscal en el contexto social contemporáneo. Revista Publicando, 3(9), 611-619.

Mejía, G. (2014). Estudio comparativo entre la legislación de eficiencia energética de Colombia y España. Revista Escuela de Administración de Negocios, 77, 122-134.

Moreno, P. (2010). Importancia de establecer un acuerdo para evitar la Doble Tributación México-España. Universidad Autónoma del Estado de Hidalgo. https://repository.uaeh.edu.mx/bitstream/handle/123456789/11731

Ortiz, N. (2015). Factores que determinan el incumplimiento de las obligaciones tributarias del impuesto general a las ventas en empresas de servicios de hotelería en el distrito de Cajamarca: Año 2013. Universidad Nacional de Cajamarca. http://repositorio.unc.edu.pe/handle/UNC/1459

Ribes, A. (2010). La tributación sucesoria de los no residentes en España a la luz de la doctrina del Tribunal de Justicia de las Comunidades Europeas. Revista Técnica Tributaria, 88, 93-117 
Rosero, A. (2011). Medición e impacto de la actividad económica informal en el ámbito tributario en la ciudad de Guayaquil. RETOS. Revista de Ciencias de la Administración y Economía, l(2), 69-78. https://www.redalyc.org/articulo.oa?id=504550952005

Sánchez, E. Fernández, F. (2018). La relación entre la política tributaria y el control del tabaquismo en Cuba. Correo Científico Médico de Holguín, 22(2), 238-249.

Sánchez, G. B. (2018). Causas de la evasión tributaria en los comerciantes mayoristas del sector abarrotes en el mercado la Hermelinda de Trujillo, 2017. Revisión de la Literatura. Universidad Privada del Norte. http://hdl.handle.net/11537/14609.

Sandoval, C. Ormazabal, M. (2017). Economía circular. Memoria Investigaciones en Ingeniería, $15,85-95$.

Wulff, E. (2008). El paradigma del acceso libre a la gestión de la información científica en $\begin{array}{lllll}\text { ciencias marinas. } & \text { Liinc }\end{array}$ https://digital.csic.es/handle/10261/7400

\section{Conflicto de intereses / Competing interests:}

Los autores declaran que no incurren en conflictos de intereses.

Rol de los autores / Authors Roles:

Edgar Callohuanca: conceptualización, curación de datos, análisis formal, adquisición de fondos, investigación, metodología, administración del proyecto, recursos, software, supervisión, validación, visualización, escritura preparación del borrador original, escritura - revisar \& amp; edición.

Ludwing Flores: conceptualización, análisis formal, investigación, metodología, administración del proyecto, recursos, software, supervisión, validación, visualización, escritura - preparación del borrador original, escritura revisar \& amp; edición.

Wilson Sucari: conceptualización, investigación, metodología, administración del proyecto, recursos, software, supervisión, validación, visualización, escritura - preparación del borrador original, escritura - revisar \& amp; edición.

Fuentes de financiamiento / Funding:

Los autores declaran que no recibieron un fondo específico para esta investigación.

\section{Aspectos éticos / legales; Ethics / legals:}

Los autores declaran no haber incurrido en aspectos antiéticos, ni haber omitido aspectos legales en la realización de la investigación. 